\title{
Identifying Models Using Recorded Data
}

J.V. Ringwood, J. Davidson, S. Giorgi

\author{
Centre for Ocean Energy Research, Maynooth University, Maynooth, Ireland
}

\subsection{INTRODUCTION AND FUNDAMENTAL PRINCIPLES}

The modelling approach presented in this chapter is that of system identification, where models are determined from input/output data measured from the system under study (Ljung, 1999). System identification, which can be considered to be the dynamical equivalent of static function approximation, has its origins in the methods developed by Legendre, Laplace, and Gauss in the 19th century, which were used to fit functional forms to (typically) astronomical data (Nievergelt, 2000). However, since the 1960s, the field of dynamical system identification has been consolidated and many successful applications have been reported using a wide variety of methods across a broad range of application areas (Ljung, 2010).

The identification procedure is based on a sequence of steps. First a parametric structure of the model is chosen. Next a suitable input signal is applied to excite the system and the response measured. Then, using the recorded input and output signals, an identification algorithm is used to determine the optimal model parameters, which minimize some error metric between the measured output and the output predicted by the identified parametric model.
Adopting a system identification approach offers considerable flexibility, in both linear and nonlinear model parameterization, regarding the relationship to physical quantities and the desired complexity/fidelity trade-off. So called white- and grey-box models present the significant benefit of a structure well related to physical aspects of the system and the model variables usually represent physical quantities. As the shade of grey gets darker, the connection with the physical world diminishes, until the only connection of black-box models with the physical world is the representation of the overall model input and output.

Fig. 7.1 illustrates the concept of model identification from recorded wave energy converter (WEC) data. The three main ingredients to system identification, the input/output data, parametric model structure, and the identification algorithm, are each analysed in different sections of this chapter. Section 7.2 details the generation of data for the system identification process and outlines the requirements of the data to ensure that representative models are obtained. It compares the different options available to acquire the data, either from open ocean tests, physical wave tank experiments or numerical wave tank (NWT) simulations, and then outlines a range of experiments available 


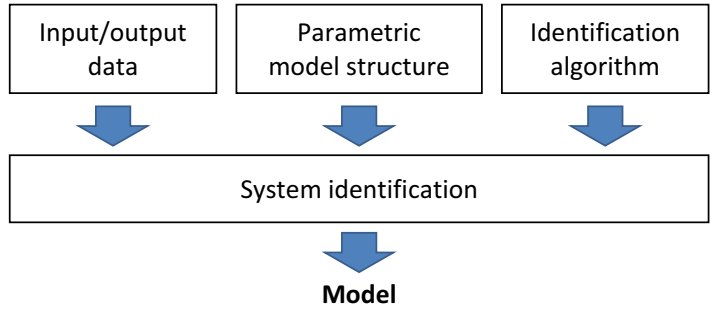

FIG. 7.1 Schematic of procedure to obtain models from recorded WEC data.

to produce the identification data for different model structures. Section 7.3 details the models for system identification. Different parametric model structures are presented, beginning with grey-box models derived from first principles and moving onto discrete-time black-box model structures. The identification algorithms are discussed in Section 7.4, by first presenting the general principles and then outlining the use of linear and nonlinear optimization methods for tuning the model parameters to fit the identification data. A number of case studies are presented in Section 7.5, giving illustrative examples of the methods described in this chapter. The limitations of this modelling approach are discussed in Section 7.6 and then a summary of the chapter given in Section 7.7.

\subsection{DATA GENERATION}

One major difficulty in system identification is ensuring that the input/output data used to determine the model is sufficiently representative of the system dynamics and, in particular, must cover the range of frequencies and amplitudes likely to be encountered during system operation. In the WEC case, such a range of excitation signals is not likely to be available in the open ocean (at least not in a reasonably short time frame) and there are difficulties in exactly enumerating the excitation experienced by the device, particularly for a directional device. In short, there is no external control of the excitation. Alternatively, it is possible to employ tank tests. However, in addition to the significant cost and the need for a physical prototype, there may be limitations on the range of excitation signals available and tank wall reflections may limit the range and duration of viable tests. One other possibility for generating suitable input/output data is to use a NWT.

A NWT is the generic name of numerical simulators for modelling nonlinear free surface waves, hydrodynamic forces and floating body motions (Tanizawa, 2000). Current day computing power allows the implementation of NWTs using computational fluid dynamics (CFD), which is described in detail in Chapter 6 . Using a CFD based NWT to generate data for WEC model identification has the following advantages:

- Reflections from 'tank' walls can be effectively controlled,

- Can test the device at full scale, eliminating scaling effects,

- A wide variety of excitation signals, including incident waves and forces directly applied to the device, as well as free response tests, can be implemented,

- The device can be constrained to different modes of motion without requiring mechanical restraints, which can add friction and alter the device dynamics,

- Hydrodynamic force measurement,

- Signals can be passively measured without requiring physical sensor devices, which can alter the device or fluid dynamics and are subject to measurement error, and most importantly,

- Specialist equipment, including a prototype WEC device, is not required.

However, NWTs are not without their drawbacks. The chief disadvantage, over a conventional wave tank, is the excessively long time to perform the numerical computation of the response, and typical computation times can be up to 1000 times the simulation time: ie, $1 \mathrm{~s}$ 
of simulation time takes $1000 \mathrm{~s}$ to compute. In addition, NWTs can take considerable experience to use well, in particular setting up a spatial mesh, which offers a reasonable compromise between computation time and accuracy. The accuracy of an NWT simulation is difficult to assess without experimental validation, and can be limited by numerical diffusion, which is another drawback.

The modelling method presented in this chapter directly addresses the computational complexity issue by using NWTs to develop sets of representative responses, which can subsequently be used to produce computationally efficient parametric models. The construction of these system identification tests needs to be carefully considered, so that representative input/output behaviour over the operational space is recorded, while minimizing overall computation time.

The use of CFD based NWTs to identify hydrodynamic parameters has been reported by a number of researchers this decade. For example, Bhinder et al. (2011) used CFD experiments to identify a coefficient for a viscous damping term to be added to the Cummins equation. Bonfiglio et al. (2011) performed prescribed harmonic oscillation experiments to determine the added mass and radiation resistance coefficients of a floating body at discrete frequencies. Davidson et al. (2013) used free decay experiments to identify the full state dynamics of a floating body. Armesto et al. (2014) used free decay experiments and input waves to identify the state plus input dynamics of an oscillating water column. Davidson et al. (2014) identified the state plus input dynamics of a floating body, by introducing a power take-off (PTO) force to the body to drive its motion and also determined nonlinear hydrodynamic restoring force parameters from the NWT experiments. Giorgi et al. (2015) used input waves to identify nonlinear hydrodynamic excitation force kernals. Ringwood et al. (2015) details optimizing
NWT experiments for the identification of hydrodynamic models and shows examples of both input waves and PTO forces in a NWT experiment to train and validate a generalized hydrodynamic model.

\subsubsection{Identification Experiments}

This section details the various experiments available to produce data to identify the parameters of WEC models. The different tests are used to identify different types of models, or submodel components, depending on their required input/output signals.

\subsubsection{Free Decay}

In a free decay experiment, a body is initially displaced from equilibrium against a restoring force and the resulting body motion simulated, shown in Fig. 7.2A. For the heave, pitch, and roll modes of motion, the mismatch between the gravitational and buoyancy forces acts as the restoring force. The surge, sway, and yaw modes have no natural restoring forces; however, a spring force can be applied to the body, to allow free decay experiments to be performed for these modes of motion.

There is no input to the system in the free decay test, therefore the data can be used to identify the system's state dynamics separately (see Case study 1 in Section 7.5.1). The free decay experiment can also be used as a preliminary test to give an indication of the system's natural frequency and bandwidth, shown in Fig. 7.2B, which can be used to inform the design of subsequent identification experiment's input frequency range.

\subsubsection{Input Waves}

For the input waves experiments, waves are created and the free surface elevation (FSE), $\eta$, is measured at a location and used as the input signal for the model identification. Depending on the model to be identified, there are two different output options for these experiments, 

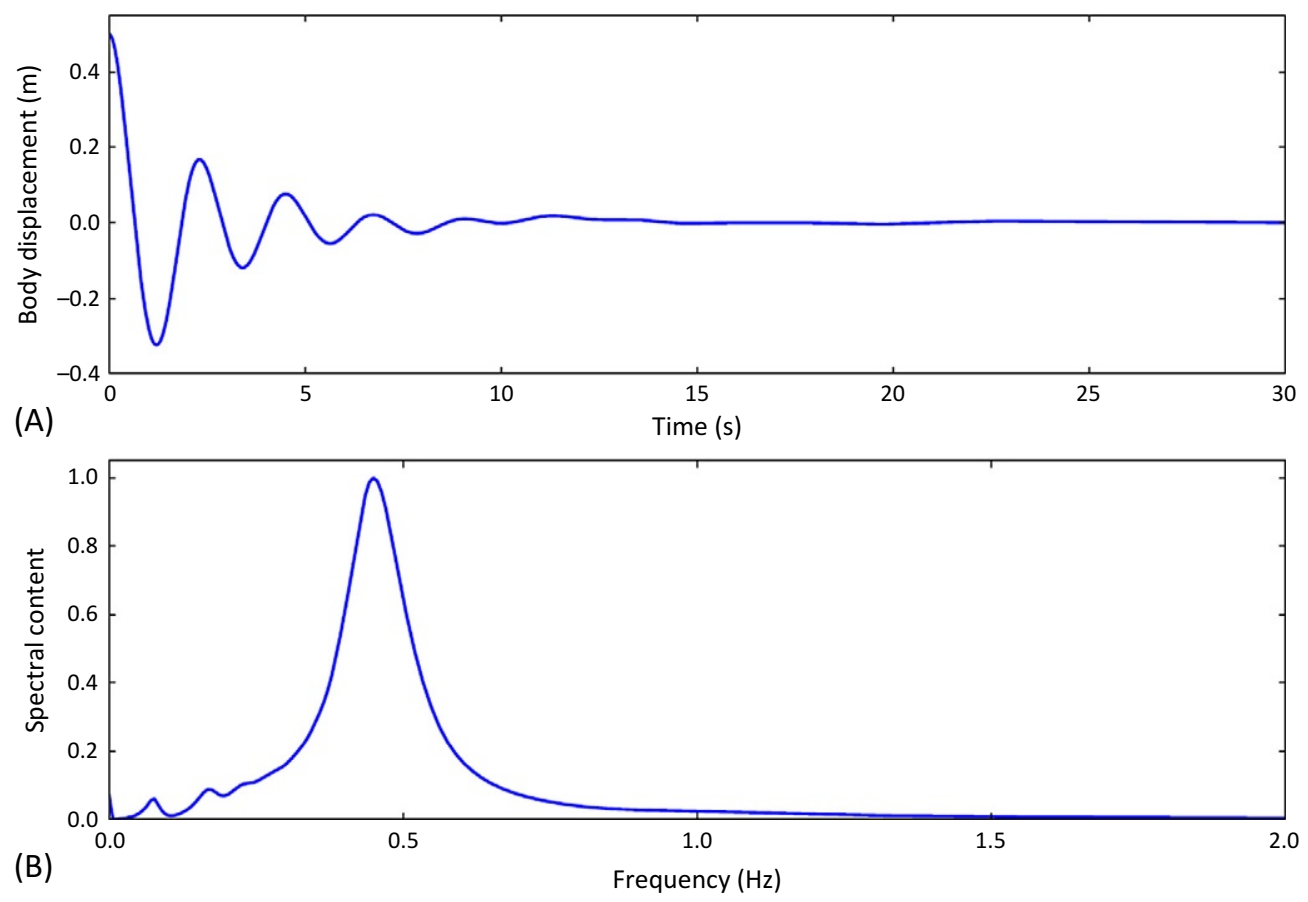

FIG. 7.2 Device free response in (A) time, and (B) frequency domains.

either body motion or excitation force. For body motion, the WEC can be constrained to different degrees of freedom if required and the resulting position and/or velocity is measured and used as the output signal for the model identification. For the excitation force, the body is held fixed and the force from the fluid on the body is measured and used as the output signal for the identification of the excitation force kernal.

Often, the required input to the model is the FSE at the WEC's centre of mass. In this case, it is not possible to directly measure this quantity since the body occupies that space. A possible solution is the employment of techniques of spatial reconstruction of the wave field, using a group of sensors located in the proximity of the floating body. Alternatively, the experiment can be decomposed into two separate experiments, where: firstly the input waves are generated and measured in the absence of the WEC (Fig. 7.3A), and then, the experiment is repeated with the same input waves being generated with the WEC in place (Fig. 7.3B). An example of the input FSE and resulting body motion are shown in Fig. 7.3C.

The frequency and amplitude range for these experiments is limited by the underlying fluid dynamics and limiting wave steepness. The choice of input signals should be guided by the sea-states the WEC is expected to operate in. The experiments can be designed using either specific individual sea spectrums, or by creating a generalized broadbanded spectrum spanning the entire set of expected spectrums likely to be encountered at a location. Monochromatic waves are also a possibility if desired.

\subsubsection{Input Force}

In an input force experiment, an external force/torque is directly applied to the body as input, the resulting motion measured as output and the input/output data then used to identify 

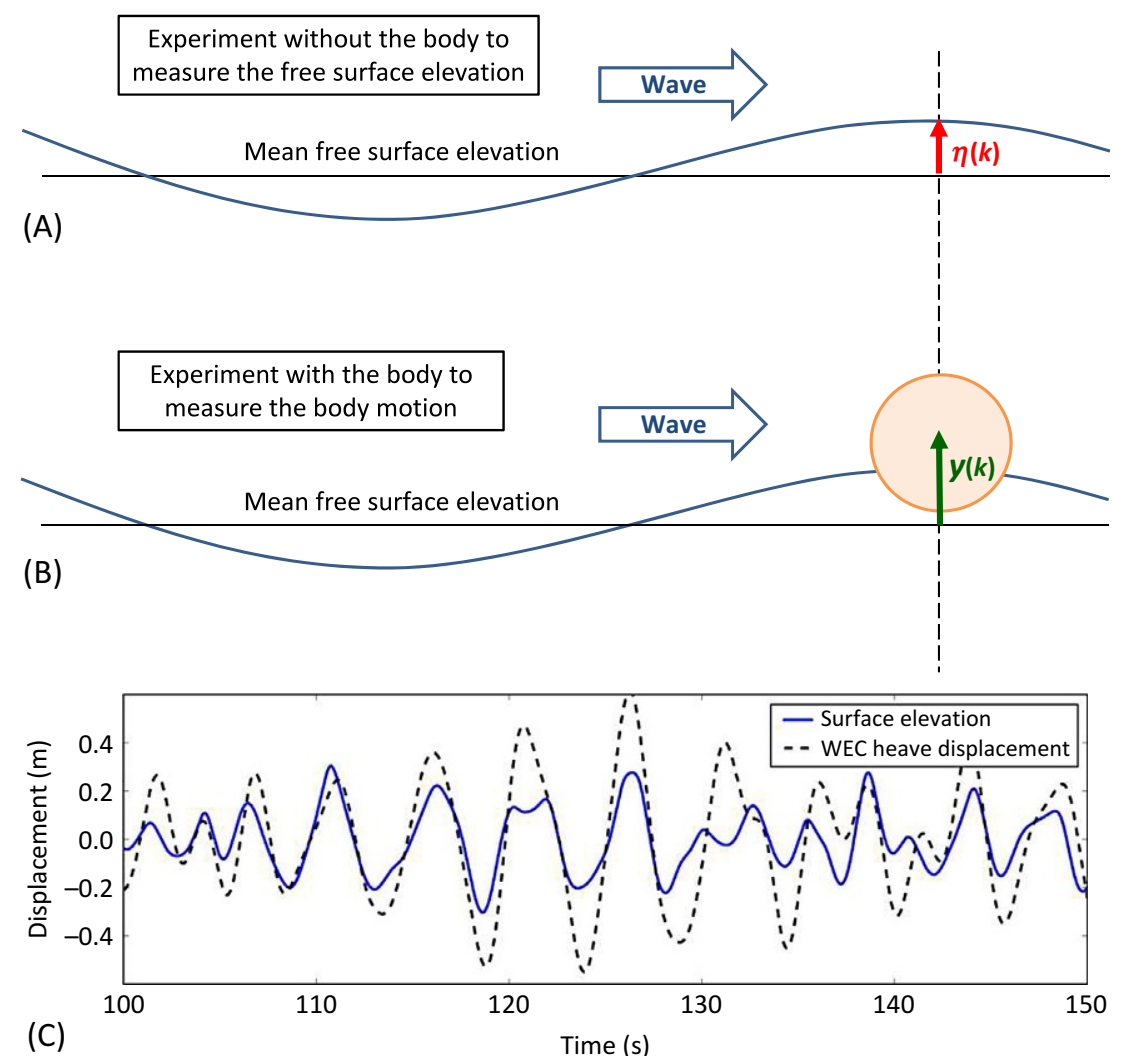

FIG. 7.3 Input waves experiment. (A) The input waves are generated and measured without the body. (B) The experiment is repeated with the same input waves being generated with the body. (C) Example of FSE and body displacement.

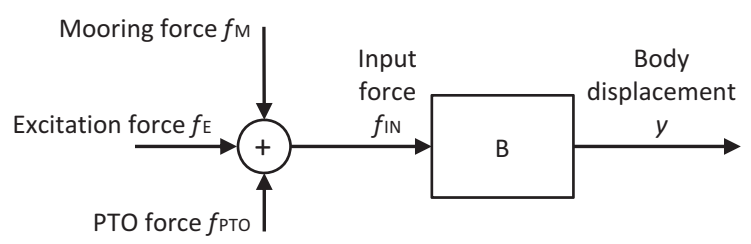

FIG. 7.4 Block diagram for the input force to body motion model.

model structures such as those shown in Fig. 7.4. This model structure is based on the superposition of input forces to the body, which for a WEC could be: the excitation force from the waves, $f_{\mathrm{E}}$, the force from the PTO, $f_{\text {РTO }}$, and the force from the mooring, $f_{\mathrm{M}}$. Case study 2 in Section 7.5.2 gives an example of identifying this type of model structure from input force experiment data.

Unlike the input waves experiment, where the choice of input signal is constrained by the laws of fluid dynamics, the input force experiment allows total freedom over the choice of input signal to be applied to the system. For linear systems, the requirement on the input signal is simple: make sure that the input signal covers the frequency range of interest. This could be ascertained from either some rough measurement of the resonant frequency and bandwidth of the device, or the range of sea frequencies in which the WEC is expected to operate. Usually these two frequency ranges are well connected; however, the device may also be subjected to 
high-frequency inputs from mooring snap loads, maximum stroke end-stop collisions, latching control, etc.

Typically, useful signals for the identification of linear systems are pseudo-random binary sequences, which have a flat frequency spectrum, or multi-sinusoids, which contain a set of closely packed frequencies. For nonlinear systems, there is an additional input/output signal amplitude dependence in the model, so the full operational range of amplitudes will also, ideally, need to be present in the identification signal. Therefore, pseudo-random sequences with randomly varying amplitudes (or random amplitude, random period [RARP]) can be employed for nonlinear systems. Chirp and multisine signals are also useful, since the input signal varies continuously over the allowable input amplitude range (Pintelon and Schoukens, 2012). Examples of these different input signal possibilities are shown in Fig. 7.5, and an analysis of their different attributes for identifying generalized hydrodynamic models presented in Ringwood et al. (2015).
Fig. 7.6 shows the output body motion from an input chirp signal force, which linearly sweeps the frequency range $0-2 \mathrm{~Hz}$ (top axis) in $600 \mathrm{~s}$ (bottom axis). The response is shown in Fig. 7.6 for the same device shown in Fig. 7.2, where both figures show a resonant peak around $0.45 \mathrm{~Hz}$. This type of experiment can be useful to gauge the bandwidth of the device's total forced dynamics (state plus input), and, unlike the chirp signal used for the later identification experiments, which must cover a desired amplitude range, the chirp signal in this preliminary experiment can be of very low amplitude, allowing fast simulation times in the cases where an NWT is being used.

\subsubsection{Prescribed Motion}

In a prescribed motion experiment, the body moves along a predefined trajectory, while the resulting force from the fluid is recorded. For this type of experiment, the motion of the body is the input and the hydrodynamic force on the body is the output. These types of experiments
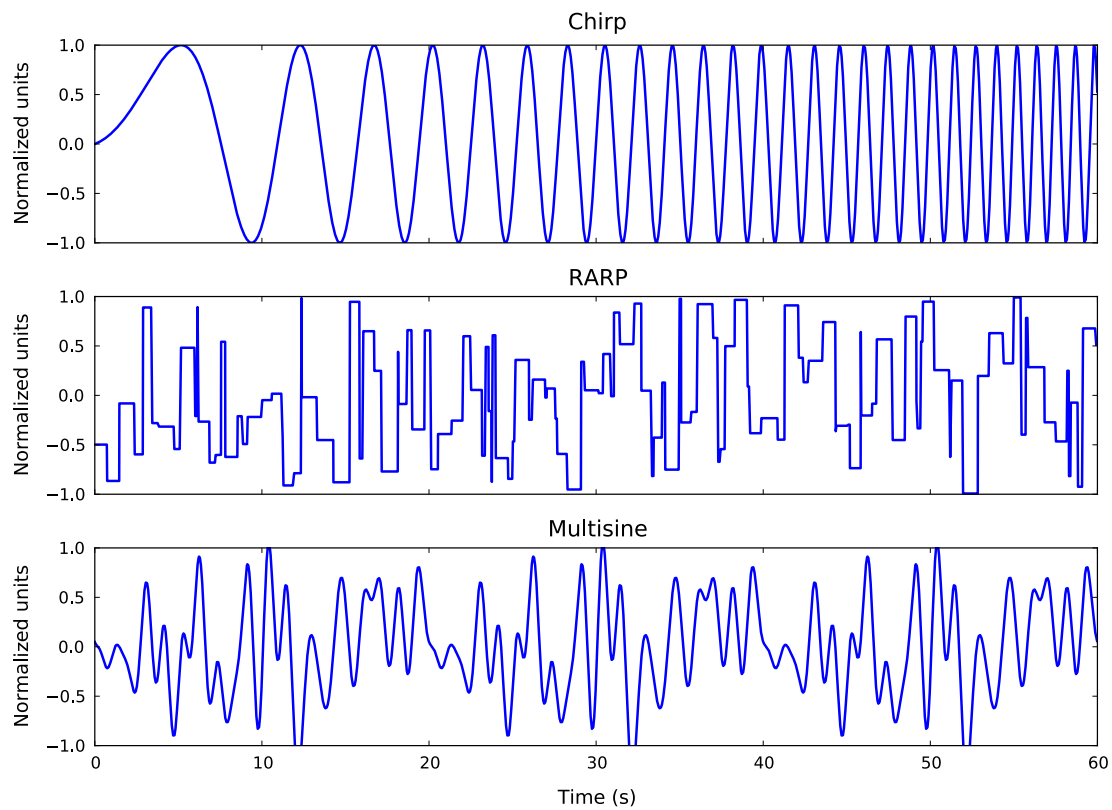

FIG. 7.5 Example input force signal possibilities. 


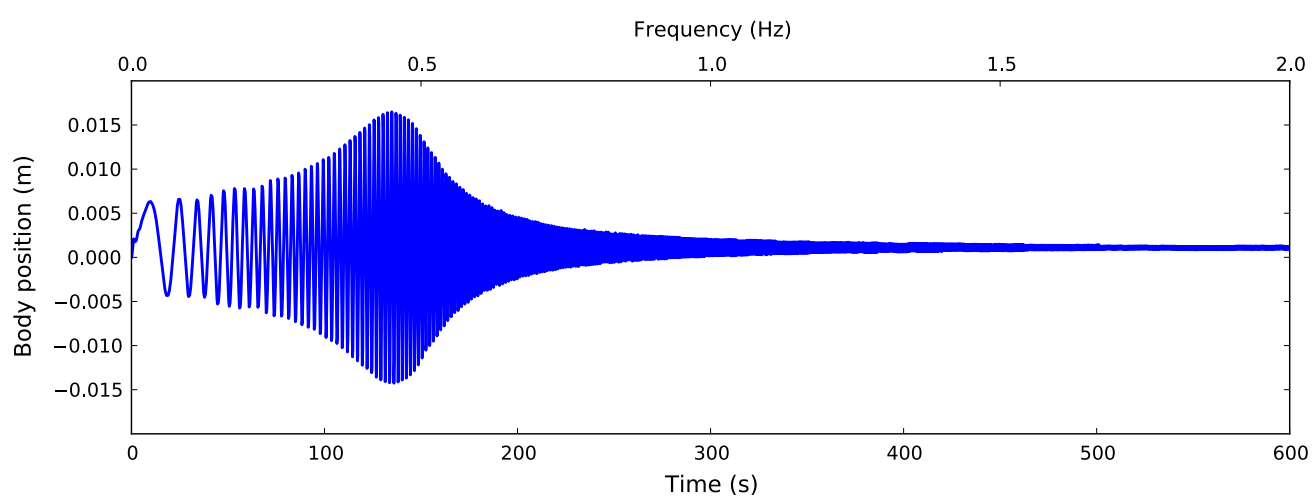

FIG. 7.6 Body motion output from input chirp signal.

can be used to identify radiation and restoring force parameters. Fig. 7.16 in Case Study 2 shows an example of a prescribed motion experiment, designed to measure the hydrostatic restoring force for a WEC.

\subsection{MODELS FOR SYSTEM IDENTIFICATION}

The choice of the model parametric structure is key if a representative model is to be determined. Hydrodynamic models with a linear input/output relationship have very desirable properties, such as superposition or a frequency domain description, but they are based on the hypothesis of small waves, ideal fluid (inviscid and incompressible) and small body displacement, which are not usually satisfied in reality. Consequently, when the wave amplitude and the body displacement increase (becoming of the same order of magnitude of the dimensions of the body), some nonlinear effects may appear (viscosity, nonlinear restoring, nonlinear FroudeKrylov force, etc.). To avoid the reduction of model performances, some nonlinearities have to be introduced into the model structures. In recent years, a relatively large number of continuous-time (CT) models have been developed (Section 7.3.1), but, in Section 7.3.2, a family of discrete-time (DT) models are proposed as a new alternative for WEC modelling.

In general, model structures for system identification usually contain terms to handle the effects of measurement noise (Ljung, 1999). Measurement noise arises due to the inherent uncertainty in trying to measure a physical quantity in the real world. Consequently, the recorded data, used to identify the model parameters, contains both the useful system information as well as disturbances from the noise, and the model structure is adapted to handle this fact. However, for data generated in an NWT, no measurement error is involved, which therefore significantly simplifies the structure of the identified model, by eliminating the requirement of additional terms to account for the noise. For simplicity, the models described in this chapter assume NWT generated data; however, they can be extended for the case of physical tank and open ocean datasets using the methods described in Ljung (1999).

\subsubsection{Continuous-Time Models}

Continuous-time models for WEC are typically based on Newton's law of motion and the decomposition of the fluid force into the 
excitation, radiation, and restoring forces. Once the parametric model structures are obtained, the parameters can be identified from available data. Chapter 2, utilizing potential flow theory and boundary element methods (BEM), describes the generation of nonparametric results in the frequency domain, which can be utilized for CT linear hydrodynamic model identification. Chapter 3 describes different model structures based on the Cummins equation in the time domain. The identification of linear hydrodynamic models is not restricted to data generated with BEM; indeed, it is possible also to employ data from physical wave tanks or from NWT (Davidson et al., 2015b; Armesto et al., 2014). The linear models can be enriched introducing nonlinear terms able to describe specific nonlinear physical effects, such as viscosity (Bhinder et al., 2012), nonlinear FroudeKrylov force (Babarit and Laporte-Weywada, 2009; Guérinel et al., 2011; Lawson et al., 2014) or nonlinear restoring force (Zurkinden et al., 2014).

\subsubsection{Discrete-Time Models}

Considering the discrete-time nature of sampled data from experiments, the majority of system identification techniques are based on discrete-time models (Ljung, 1999). Discretetime modelling uses signals only specified at the discrete time instants $t=k T_{\mathrm{s}}$, where $T_{\mathrm{s}}$ is the sampling period and $k$ is an integer. For example, the values of $\eta, f_{I N}$, and $y$ at the time instant $k T_{\mathrm{s}}$ are represented with the symbols $\eta(k), f_{I N}(k)$, and $y(k)$, respectively.

In this chapter, nonlinear autoregressive with exogenous input (NARX) models (Nelles, 2001) are utilized. In NARX models, the present value of the output $y(k)$ depends on the past $n_{a}$ values of the output $y(k-1), \ldots$, $y\left(k-n_{a}\right)$ and the $n_{b}+1$ input values $u\left(k-n_{d}\right)$, $u\left(k-n_{d}-1\right), \ldots, u\left(k-n_{d}-n_{b}\right)$. If the system is causal, the output does not depend on future values of the input and $n_{d} \geq 0$; otherwise, if the system is noncausal, $n_{d}<0$ and the present value of the output is influenced by future input values. The NARX model is summarized with the following equation:

$$
\begin{aligned}
y(k)= & g\left[y(k-1), \ldots, y\left(k-n_{a}\right), u\left(k-n_{d}\right),\right. \\
& \left.u\left(k-n_{d}-1\right), \ldots, u\left(k-n_{d}-n_{b}\right)\right],
\end{aligned}
$$

which can be represented with the block diagram of Fig. 7.7. $n_{a}$ and $n_{b}$ represent the dynamical order of the model, and by increasing them, the model becomes more flexible and able to show more complex dynamical behaviour but, at the same time, unnecessarily high orders can make the model less able to generalize on new data (overfitting). $n_{d}$ is the input delay time and it represents the number of samples before the output reacts to the input (for $n_{d} \geq 0$ ) or the number of future input steps that influence the present value of the output (for $n_{d}<0$ ).

A number of different types of NARX models are described in this section, which can be obtained by changing the function $g()$ in Eq. (7.1), namely: the autoregressive with exogenous input (ARX) model, the KolmogorovGabor polynomial (KGP) model, the artificial

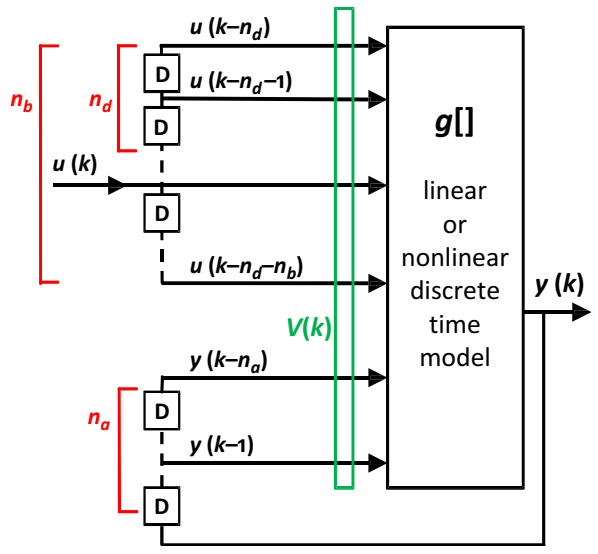

FIG. 7.7 Block diagram for a general NARX discrete-time model. Each D-block denotes a single delay element. $n_{a}, n_{b}$, and $n_{d}$ indicate the number of D-blocks. 
TABLE 7.1 Properties Overview of the ARX, KGP, ANN, NLS, and BONL models

\begin{tabular}{llllll}
\hline Property & ARX & KGP & ANN & NLS & BONL \\
\hline $\begin{array}{l}\text { Input/output } \\
\text { relationship }\end{array}$ & L & NL & NL & NL & NL \\
Optimization & L & L & NL & L & L \\
Shade of grey & B & B & B & G & G \\
Memory & D & D & D & S & D \\
\hline
\end{tabular}

$L$, linear; $N L$, nonlinear; $B$, black-box; $G$, grey-box; $D$, dynamic; and $S$, static.

neural network (ANN), the nonlinear static (NLS) model, and the block-oriented nonlinear (BONL) model. An overview of the different models' properties is shown in Table 7.1. For more details regarding these models see Nelles (2001) and Giri and Bai (2010).

\subsubsection{Autoregressive With Exogenous Input Model (Linear)}

The first discrete-time model presented is the autoregressive with exogenous input model. It is a well known black-box model with a linear input/output relationship and it is linear in the parameters $a_{i}$ and $b_{i}$. For the ARX model, Eq. (7.1) becomes:

$$
y(k)=\sum_{i=1}^{n_{a}} a_{i} y(k-i)+\sum_{i=0}^{n_{b}} b_{i} u\left(k-n_{d}-i\right) .
$$

Advantages of the ARX model:

- Linear in the parameters (fast identification with guaranteed global minimum),

- Linear input/output relationship (superposition and a frequency domain description).

Disadvantages of the ARX model:

- Linear input/output relationship (it cannot capture nonlinear behaviours),

- Black-box (parameters have no physical meaning).

Examples of ARX models are shown in the case studies in Sections 7.5.2 and 7.5.3.

\subsubsection{Kolmogorov-Gabor Polynomial Model (Nonlinear)}

The KGP model utilizes a polynomial nonlinearity to describe the input/output relationship (Nelles, 2001). In this case, Eq. (7.1) becomes:

$$
\begin{aligned}
y(k)= & \sum_{i=1}^{n_{a}} a_{i 1} y(k-i)+\sum_{i=0}^{n_{b}} b_{i 1} u\left(k-n_{d}-i\right)+\cdots \\
& +\sum_{i=1}^{n_{a}} a_{i p} y^{p}(k-i)+\sum_{i=0}^{n_{b}} b_{i p} u^{p}\left(k-n_{d}-i\right) \\
& +\sum_{i=1}^{n_{a}} \sum_{j=0}^{n_{b}} c_{i j} y(k-i) u\left(k-n_{d}-j\right)+\cdots
\end{aligned}
$$

where $p$ is the maximum polynomial order for the terms involving $u$ and $y$ alone, as well as the cross-product terms. The KGP model is a black-box model having a nonlinear input/ output relationship, but is linear in the parameters $a_{i j}, b_{i j}$, and $c_{i j}$. An example of a KGP model is shown in Case Study 3 in Section 7.5.3.

Advantages of the KGP model:

- Linear in the parameters (fast identification with guaranteed global minimum),

- Nonlinear input/output relationship (able to capture nonlinear behaviours).

Disadvantages of the KGP model:

- Nonlinear input/output relationship (no superposition and no frequency domain description),

- Black-box (parameters have no physical meaning).

\subsubsection{Artificial Neural Network Model (Nonlinear)}

The multilayer perceptron (MLP) artificial neural network, composed by input, hidden, and output layers (Nelles, 2001), is a black-box model having a nonlinear input/output relationship and is nonlinear in the parameters. While one hidden layer of nonlinear neurons 
can give an arbitrary approximation capability, two hidden layers usually requires a lower total neuron count, for a given level of approximation accuracy. The overall model complexity is determined by $n_{1}$ and $n_{2}$, the number of neurons in the hidden layers 1 and 2, respectively, in addition to $n_{a}$ and $n_{b}$. The feedforward MLP is defined by $y(k)=g[V(k)]$, as in Fig. 7.7, where

$$
\begin{aligned}
V(k)= & {\left[v_{1}(k), v_{2}(k), \ldots, v_{n_{v}}(k)\right] } \\
= & {\left[y(k-1), \ldots, y\left(k-n_{a}\right), u\left(k-n_{d}\right),\right.} \\
& \left.u\left(k-n_{d}-1\right), \ldots, u\left(k-n_{d}-n_{b}\right)\right]
\end{aligned}
$$

$n_{v}=n_{a}+n_{b}+1$ is the number of variables. In this way, the input/output relationship of the ANN model can be written (Nelles, 2001) as:

$$
y(k)=\sum_{i=0}^{n_{2}} w_{i}^{(\text {out })} \Psi_{i}\left(\sum_{j=0}^{n_{1}} w_{i j}^{(2)} \Psi_{j}\left(\sum_{l=0}^{n_{v}} w_{j l}^{(1)} v_{l}(k)\right)\right)
$$

where $v_{0}(k)=1, w_{j l}^{(1)}, w_{i j}^{(2)}$, and $w_{i}^{(\text {out })}$ are the unknown parameters of hidden layer 1 , hidden layer 2, and the output layer, respectively, and $\Psi_{i}$ is the activation function. We choose tansigmoidal activation functions, which provide global support and give a smooth interpolation between training data points, defined as:

$$
\Psi_{i}(z)= \begin{cases}1 & \text { if } i=0 \\ 2 /\left(1+e^{-2 z}\right)-1 & \text { if } i \neq 0\end{cases}
$$

Advantages of the ANN model:

Nonlinear input/output relationship (able to capture nonlinear behaviours).

Disadvantages of the ANN model:

- Nonlinear input/output relationship (no superposition and no frequency domain description),

- Nonlinear in the parameters (difficult optimization with many local minima), - Black-box (parameters have no physical meaning).
An example of an ANN is shown in Case Study 3 in Section 7.5.3.

\subsubsection{Nonlinear Static Model (Nonlinear)}

A simple way to model a nonlinear relationship between the input $u(k)$ and the output $y(k)$ of a model is to utilize a nonlinear static block $r$, obtaining $y(k)=r(u(k))$. In this case, the model has no memory and the output at the instant $k$ depends only on the input at the same instant $k$. The static function $r$ can be approximated with a linear combination of basis functions $\left\{\zeta_{1}, \zeta_{2}, \ldots, \zeta_{n_{c}}\right\}$, obtaining:

$$
y(k)=\sum_{i=1}^{n_{c}} c_{i} \zeta_{i}(u(k)) .
$$

Advantages of the NLS model:

- Linear in the parameters (fast identification with guaranteed global minimum),

- Nonlinear input/output relationship (able to capture nonlinear behaviours).

Disadvantages of the NLS model:

- Nonlinear input/output relationship (no superposition),

- No memory (not able to capture dynamic behaviours).

\subsubsection{Block-Oriented Nonlinear Model (Nonlinear)}

Connecting linear dynamic ARX blocks and nonlinear static blocks in different number and configuration, it is possible to obtain a variety of nonlinear dynamic model structures (Hammerstein model, Wiener model, etc.) (Giri and Bai, 2010). The obtained models are called BONL models.

As an example, the very well known Hammerstein model consists of a cascade connection of a nonlinear static block followed by a linear dynamic block (see Fig. 7.8A). In the Hammerstein case, Eq. (7.1) becomes (Pearson and Pottmann, 2000): 


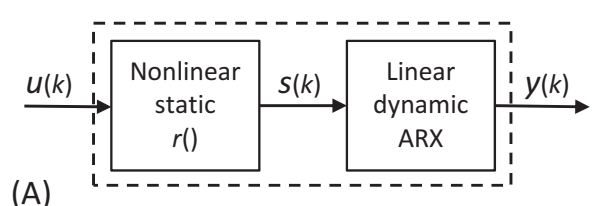

(A)

(B)

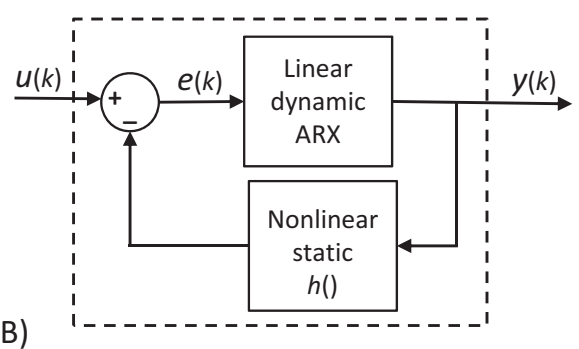

FIG. 7.8 (A) Block diagram of the Hammerstein model. (B) Block diagram of the feedback block-oriented model.

$$
y(k)=\sum_{i=1}^{n_{a}} a_{i} y(k-i)+\sum_{i=0}^{n_{b}} b_{i} r\left(u\left(k-n_{d}-i\right)\right)
$$

where $r()$ is a nonlinear static function. The Hammerstein model is characterized by a nonlinear input/output relationship (if $r$ is not linear), but is linear in the parameters $a_{i}$ and $b_{i}$.

In the Hammerstein model, the static relationship between input and output is given by the product of the static function, $r()$, and the steady-state gain of the ARX model, $D C_{\text {gain }}$. Therefore, the characterization of the two blocks is not unique; any pair $\left(D C_{\text {gain }} / \alpha, \alpha r\right)$, where $\alpha$ is an arbitrary constant, will produce the same input and output (Bai, 2003). To remove this ambiguity, the $D C_{\text {gain }}$ of the ARX block is generally set to unity, which allows the steady-state gain for the entire Hammerstein model to be solely represented by the nonlinear static function. An example of Hammerstein model application is presented in Case Study 2 in Section 7.5.2.

Another example of BONL models is the feedback block-oriented model, shown in Fig. $7.8 \mathrm{~B}$, and it is characterized by a feedback nonlinear static block, $h()$, in between output and input. The equations that describe the relationship between input and output are:

$$
y(k)=\sum_{i=1}^{n_{a}} a_{i} y(k-i)+\sum_{i=0}^{n_{b}} b_{i} e\left(k-n_{d}-i\right),
$$

where

$$
e(k)=u(k)-h(y(k)) .
$$

An example of a feedback block-oriented model is presented in Case Study 2 in Section 7.5.2.

Advantages of BONL models:

- Linear in the parameters (fast identification with guaranteed global minimum),

- Nonlinear input/output relationship (able to capture nonlinear behaviour),

- It decomposes the model into submodels (divide and conquer).

Disadvantages of BONL models:

- Nonlinear input/output relationship (no superposition and no frequency domain description).

\subsection{IDENTIFICATION ALGORITHMS}

This section considers the fundamental principle of system identification. The techniques of linear and nonlinear optimization are introduced, which are used for the parameter estimation of models that are linear or nonlinear in the parameters, respectively.

\subsubsection{System Identification}

The fundamental principle of system identification is captured in Fig. 7.9. The identification procedure follows the following steps:

1. A parametric structure is chosen for the model,

2. A suitable input signal, $u$, is synthesized and input to the system,

3. The input signal, $u$, and resulting output signal, $y$, are recorded 


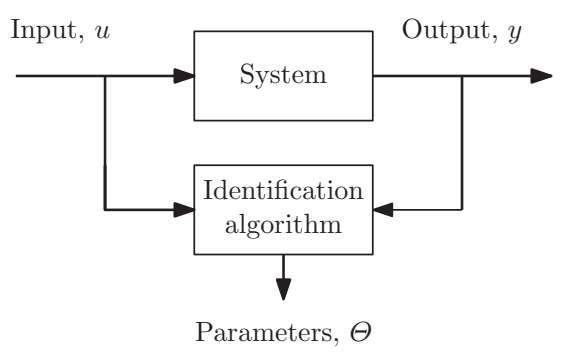

FIG. 7.9 System identification principle: the real system to be modelled generates the input data $u$ and output data $y$, which are utilized by the identification algorithm to estimate the parameters of the model.

4. An identification algorithm is used to determine the optimal parameter vector, $\Theta$, which minimizes some error metric between the actual measured output, $y$, and that produced by the identified parametric model, $\hat{y}$.

Normally, discrete-time models of the form:

$$
\begin{aligned}
y(k)= & M\left[\Theta, u(k), \ldots, u\left(k-n_{b}\right),\right. \\
& \left.y(k-1), \ldots, y\left(k-n_{a}\right)\right]
\end{aligned}
$$

are identified from samples input and output data where, in general, $M$ can be linear or nonlinear. However, identification of continuoustime models can also be performed (Rao and Unbehauen, 2006). In many cases, nonlinear models can be constructed that are linear in the parameters, easing the task of parameter identification. In general, a quadratic error metric is employed, of the form:

$$
I=\sum_{k=1}^{N}[y(k)-\hat{y}(k)]^{2} .
$$

If the model in Eq. (7.3) is linear in the parameter set $\Theta$ and a quadratic form of error metric, such as Eq. (7.4), is employed, then the optimization problem to estimate $\Theta$ is a convex one and simple optimization algorithms, such as least squares, may typically be employed. If $M$ is nonlinear, then a nonconvex optimization problem results and care must be taken to use a search algorithm that is insensitive to local minima. If the input/output measurement models contain a significant amount of noise, explicit noise models (such as ARMAX models Ljung, 1999) may be employed to avoid biased estimates. Both causal and noncausal models can be identified, so long as the requirement is not to provide model parameter estimates in real time, by appropriately shifting the input time series.

\subsubsection{Linear Optimization}

Consider a model with an output, $\hat{z}$, that is a linear combination of $q$ independent variables, $\varphi_{i}$ :

$$
\hat{z}=\theta_{1} \varphi_{1}+\cdots+\theta_{q} \varphi_{q}
$$

where the coefficients, $\theta_{i}$, are the unknown parameters (Nelles, 2001). It will be assumed that $N$ data samples are measured so that the difference at each sample between the measured data, $z$, and the model prediction is:

$$
\boldsymbol{\varepsilon}=\mathbf{z}-\hat{\mathbf{z}}=\mathbf{z}-\mathbf{X} \boldsymbol{\theta},
$$

where

$$
\begin{gathered}
\boldsymbol{\varepsilon}=\left[\begin{array}{llll}
\varepsilon(1) & \varepsilon(2) & \ldots & \varepsilon(N)
\end{array}\right]^{\mathrm{T}}, \\
\mathbf{z}=\left[\begin{array}{llll}
z(1) & z(2) & \ldots & z(N)
\end{array}\right]^{\mathrm{T}}, \\
\hat{\mathbf{z}}=\left[\begin{array}{llll}
\hat{z}(1) & \hat{z}(2) & \ldots & \hat{z}(N)
\end{array}\right]^{\mathrm{T}}, \\
\boldsymbol{\Phi}=\left[\begin{array}{cccc}
\varphi_{1}(1) & \varphi_{2}(1) & \ldots & \varphi_{q}(1) \\
\varphi_{1}(2) & \varphi_{2}(2) & \ldots & \varphi_{q}(2) \\
\vdots & \vdots & \ddots & \vdots \\
\varphi_{1}(N) & \varphi_{2}(N) & \ldots & \varphi_{q}(N) \\
& &
\end{array}\right] \\
\boldsymbol{\theta}=\left[\begin{array}{llll}
\theta_{1} & \theta_{2} & \ldots & \theta_{q}
\end{array}\right]^{\mathrm{T}} .
\end{gathered}
$$

$\boldsymbol{\Phi}$ and $\mathbf{z}$ are called the data matrix and the observation vector, respectively. In a full column rank context, the estimated parameters that minimize the least squares error are (Nelles, 2001):

$$
\hat{\boldsymbol{\theta}}=\underset{\theta}{\operatorname{argmin}}\left(\boldsymbol{\varepsilon}^{\mathrm{T}} \boldsymbol{\varepsilon}\right)=\left(\boldsymbol{\Phi}^{\mathrm{T}} \boldsymbol{\Phi}\right)^{-1} \boldsymbol{\Phi}^{\mathrm{T}} \mathbf{z} .
$$


If the parameters have to satisfy the linear equality constraints, $\mathbf{L} \boldsymbol{\theta}=\mathbf{d}$, the estimated parameters are then:

$$
\hat{\boldsymbol{\theta}}_{c}=\hat{\boldsymbol{\theta}}-\left(\boldsymbol{\Phi}^{\mathrm{T}} \boldsymbol{\Phi}\right)^{-1} \mathbf{L}^{\mathrm{T}}\left(\mathbf{L}\left(\boldsymbol{\Phi}^{\mathrm{T}} \boldsymbol{\Phi}\right)^{-1} \mathbf{L}^{\mathrm{T}}\right)^{-1}(\mathbf{L} \hat{\boldsymbol{\theta}}-\mathbf{d}),
$$

where $\hat{\boldsymbol{\theta}}$ is calculated via Eq. (7.8). An example of such linear equality constraints appeared in Section 7.3.2.5, whereby the $D C_{\text {gain }}$ of the Hammerstein model's ARX block is set to unity; for more details see Giorgi et al. (2015).

Linear optimization can be used to obtain the optimal parameter values for model structures which are linear in the model parameters, such as the ARX, KGP, NLS, and BONL models. The number of parameters to be identified is determined by the dynamical orders, $n_{a}$ and $n_{b}$, and a method to estimate the dynamical order of a given system, as well as the time delay, $n_{d}$, is described in the next subsection. The following subsection then shows the use of the linear optimization techniques to identify the model parameter values, for the specific case of a Hammerstein model as an example.

\subsubsection{Time Delay and Dynamical Order Estimation ( $\left.\mathbf{n}_{\mathbf{d}}, \mathbf{n}_{\mathbf{a}}, \mathbf{n}_{\mathbf{b}}\right)$}

An important part of the model structure selection is the choice of the delay $n_{d}$ and the dynamical orders $n_{a}$ and $n_{b}$. As Eq. (7.1) suggests, $y(k)$ is a function of $n_{a}$ output and $n_{b}+1$ input values, taken at different time instants. Once $n_{a}, n_{b}$, and $n_{d}$ are selected, it is possible to obtain the different model structures (ARX, Hammerstein, KGP, etc.) by changing the function $g()$. The time delay and dynamical orders are estimated by implementing a systematic trial and error process on several linear ARX models, with varying $n_{a}, n_{b}$, and $n_{d}$, and selecting the values which give the best model performance, as measured by a loss function (a measure of the modelling error). For each ARX model estimation, independent training, and validation data sets are utilized. At the end, the simplest ARX model able to repeat the validation data with a sufficient accuracy is selected (parsimonious model) (Ljung, 2013). The estimated $n_{a}, n_{b}$, and $n_{d}$ for the ARX model are utilized also with the other models (ANN, Hammerstein, KGP, etc.), since linear and nonlinear models share the same time delay and dynamical orders.

\subsubsection{Model Parameters Identification}

In this section, the identification of parameters for models that are linear in the parameters is illustrated. In particular, the Hammerstein model, which is composed of two different subblocks (a nonlinear static block and a linear dynamic block), is used as an example to show the identification procedure.

The nonlinear static function of the Hammerstein model can be approximated with a linear combination of basis functions; in this way it is possible to apply the linear regression for the identification. For simplicity, polynomials can be selected as basis functions $\left\{u^{0}, u^{1}, u^{2}, \ldots, u^{n_{c}}\right\}$; therefore, the relationship between the input and the output of the nonlinear static block is:

$$
\hat{s}(k)=c_{1} u(k)+c_{2} u^{2}(k)+\cdots+c_{n_{c}} u^{n_{c}}(k),
$$

where $c_{0}=0$ (it is imposed that applying a null input the output has to be zero).

The identification of the Hammerstein model is accomplished utilizing two different experiments. In the first experiment, the system is very slowly displaced (eliminating any dynamical effects) through its full amplitude range, to capture the static relationship between the input and the output signals, $\left\{u_{s}(k)\right\}$ and $\left\{y_{s}(k)\right\}$, which are utilized to identify the static block. The second experiment produces the signals $\left\{u_{d}(k)\right\}$ and $\left\{y_{d}(k)\right\}$, that are conversely characterized by rich dynamics, and are employed to identify the ARX block. This is a good example of experiments designed intentionally to identify specific model structures.

The first experiment investigates the behaviour of the Hammerstein model in nearly static conditions, in this case $s(k) \simeq y_{s}(k)$ (a consequence of the $D C_{\text {gain }}$ of the ARX being equal to 
one) and, therefore, the input/output signals of the static block are known and it is possible to identify the coefficient $c_{i}$. Indeed, Eqs (7.5), (7.6), and (7.7) become, respectively:

$$
\begin{gathered}
\mathbf{z}=\left[\begin{array}{llll}
y_{s}(1) & y_{s}(2) & \ldots & \left.y_{s}(N)\right]^{\mathrm{T}}, \\
\boldsymbol{\Phi} & =\left[\begin{array}{cccc}
{\left[u_{s}(1)\right]^{1}} & {\left[u_{s}(1)\right]^{2}} & \ldots & {\left[u_{s}(1)\right]^{n_{c}}} \\
{\left[u_{s}(2)\right]^{1}} & {\left[u_{s}(2)\right]^{2}} & \ldots & {\left[u_{s}(2)\right]^{n_{c}}} \\
\vdots & \vdots & \ddots & \vdots \\
{\left[u_{s}(N)\right]^{1}} & {\left[u_{s}(N)\right]^{2}} & \ldots & {\left[u_{s}(N)\right]^{n_{c}}}
\end{array}\right], \\
& &
\end{array}\right] \\
\hat{\boldsymbol{\theta}}_{\mathbf{H c}}=\left[\begin{array}{llll}
c_{1} & c_{2} & \ldots & c_{n_{c}}
\end{array}\right]^{\mathrm{T}} .
\end{gathered}
$$

From the second experiment, the ARX block is identified using the data signals $\left\{u_{d}(k)\right\}$ and $\left\{y_{d}(k)\right\}$. First, the input $\left\{u_{d}(k)\right\}$ is transformed through the nonlinear static block to obtain $\{s(k)\}$, using Eq. (7.10). Next, $\{s(k)\}$ and $\left\{y_{d}(k)\right\}$ are used as the input and output pair to identify the ARX parameters, $\hat{\theta}_{H_{A R X}}$, under the constraint that the DC gain of the ARX is equal to one. The first possible predicted model output is for $k=\tau+1$ (the first $\tau$ values of the output data are utilized as initial conditions), where $\tau=\max \left\{n_{a},\left(n_{b}+n_{d}\right)\right\}$. The last possible predicted model output is for $k=\tilde{N}$, where

$$
\tilde{N}= \begin{cases}N & \text { if } n_{d} \geq 0 \\ N+n_{d} & \text { if } n_{d}<0\end{cases}
$$

In this case, Eqs (7.5), (7.6), and (7.7) become:

$$
\begin{gathered}
\mathbf{z}=\left[\begin{array}{lllll}
y_{d}(\tau+1) & y_{d}(\tau+2) & \ldots & y_{d}(\tilde{N})
\end{array}\right]^{\mathrm{T}}, \\
\boldsymbol{\Phi}=\left[\begin{array}{cccccc}
y_{d}(\tau) & \ldots & y_{d}\left(\tau+1-n_{a}\right) & s\left(\tau+1-n_{d}\right) & \ldots & s\left(\tau+1-n_{d}-n_{b}\right) \\
y_{d}(\tau+1) & \ldots & y_{d}\left(\tau+2-n_{a}\right) & s\left(\tau+2-n_{d}\right) & \ldots & s\left(\tau+2-n_{d}-n_{b}\right) \\
\vdots & \ddots & \vdots & \vdots & \ddots & \vdots \\
y_{d}(\tilde{N}-1) & \ldots & y_{d}\left(\tilde{N}-n_{a}\right) & s(N) & \ldots & s\left(N-n_{b}\right)
\end{array}\right] \\
\hat{\boldsymbol{\theta}}_{\mathbf{H}_{\mathbf{A R x}}}=\left[\begin{array}{llllllll}
a_{1} & a_{2} & \ldots & a n_{a} & b_{0} & b_{1} & \ldots & b_{n_{b}}
\end{array}\right]^{\mathrm{T}},
\end{gathered}
$$

respectively. The estimated parameters are determined from Eq. (7.9).
The identification of the KGP model and the feedback block-oriented models, which are also linear in the parameters, is very similar to the one shown here for the Hammerstein model (see Davidson et al., 2014; Giorgi et al., 2015).

\subsubsection{Nonlinear Optimization}

For models that are nonlinear in the parameters $\theta$, it is not possible to use the linear regression techniques, shown in Section 7.4.2, for model parameter identification. The main idea of optimization is to minimize some cost function $I(\theta)$, which is a measure of error between the model prediction and the recorded data. Given a model nonlinear in the parameters, the cost function $I(\theta)$ may have many local optima and no analytical solution exists, which therefore requires the use of an iterative algorithm (see Fig. 7.10). At each step, the algorithm implements the equation $\theta_{k}=\theta_{k-1}+\delta_{k-1} \nu_{k-1}$, where the new parameter vector $\theta_{k}$ is calculated from the previous $\theta_{k-1}$, moving in a direction $\nu_{k-1}$ with a step size $\delta_{k-1}$. There is a large variety of algorithms for nonlinear optimization, like steepest descent, conjugate gradient, Levenberg-Marquard, etc. Selection of an appropriate nonlinear optimization algorithm is considered to be outside the scope of this chapter; the reader is pointed to Nelles (2001) for further details. Although each nonlinear optimization algorithm has distinct characteristics, a number of common issues should be considered. Firstly, because the problem is nonconvex, the solution is strongly dependent on the initial value $\theta_{0}$; therefore, different initial conditions have to be utilized to help the research of an appropriate local minima. Secondly, care must be taken to avoid overfitting to the data used in model identification. Consequently, early-stopping techniques should be utilized to halt the model identification as soon as errors in the validation data start to rise. In this chapter, nonlinear optimization is applied in the case studies in Sections 7.5.1 and 7.5.3. 
FIG. 7.10 Block diagram of the nonlinear optimization methodology to estimate parameters $\theta$.

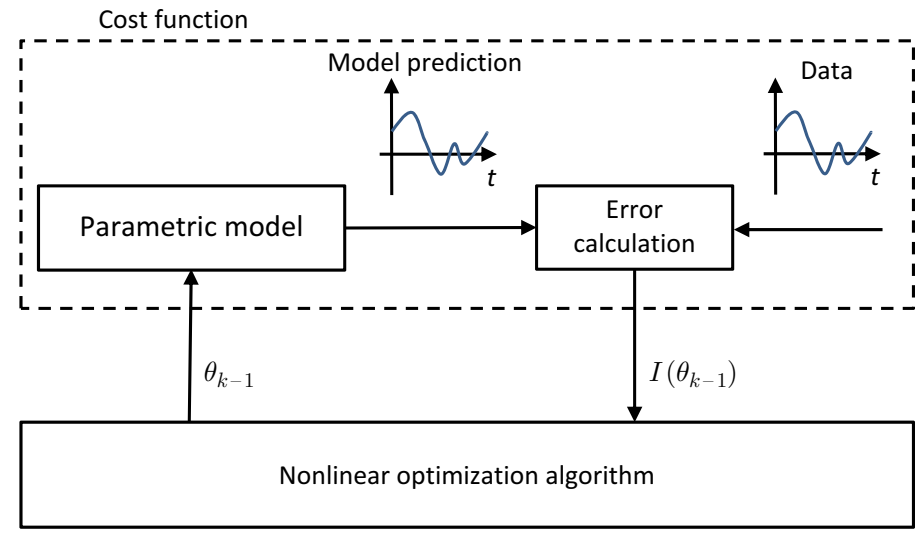

\subsection{CASE STUDIES}

Here three case studies are presented which illustrate the identification of models from recorded WEC data. The data is produced from NWT experiments using the open-source CFD software OpenFOAM. A detailed description of the implementation of this OpenFOAM NWT is presented in Davidson et al. (2015a).

\subsubsection{Case Study 1: Continuous-Time Models Identified From Free Responses}

This case study illustrates an example of linear CT model parameter identification from NWT data. The case study considers a state-space model for the heave motion of a vertical cylinder, with diameter $1 \mathrm{~m}$ and draught $0.5 \mathrm{~m}$. The parameters of the state matrix are identified from NWT data generated from free decay experiments, and are compared against results identified from the boundary element solver WAMIT. Full details for this study can be found in Davidson et al. (2015b).

Four free decay experiments with varying initial displacements of $5,10,20$, and $45 \mathrm{~cm}$, were conducted in the NWT (Fig. 7.11A). To illustrate the inherent nonlinear hydrodynamic effects captured by these CFD simulations, the results in Fig. $7.11 \mathrm{~A}$ are normalized against their initial positions and plotted in Fig. 7.11B. Linearity would require these free decay curves to overlay each other when normalized (if linear scaling applies). However, due to nonlinear effects, such as viscosity, vortex shedding, and time varying wetted body surface area, the normalized NWT responses vary for different initial amplitudes. In particular it can be seen the experiments with larger initial amplitudes have larger decay rates.

The results from the four different free decay experiments are then used to separately identify four individual state-space models. The statespace models are derived from the Cummins equation and are parameterized by $2 n+3$ parameters, namely: the body mass, $M$; the infinite frequency added-mass, $m_{\infty}$; the restoring force coefficient, $K$; and the parameters of the radiation force state-space subsystem $\left(a_{0}, \ldots\right.$, $a_{n-1}, b_{0}, \ldots, b_{n-1}$ ) (see Section 3.3.3). The body mass, $M$, is assumed known, being a required input for the NWT simulations, and can be determined from a variety of numerical modelling tools, such as AutoCAD. The restoring force coefficient can be determined as the product of the water density, $\rho$, the gravitational constant, $g$, and the cross sectional area of the cylinder, $S$, ie, $K=\rho g S$. The remaining parameters, $\theta=\left[m_{\infty}, a_{0}, \ldots, a_{n-1}, b_{0}, \ldots, b_{n-1}\right]$, are then determined using the nonlinear optimization methodology shown in Fig. 7.10, such that the model output, $y\left(t_{i}, \theta\right)$, approximates the free decay NWT data, $y_{N W T}\left(t_{i}\right)$, via the criterion: 

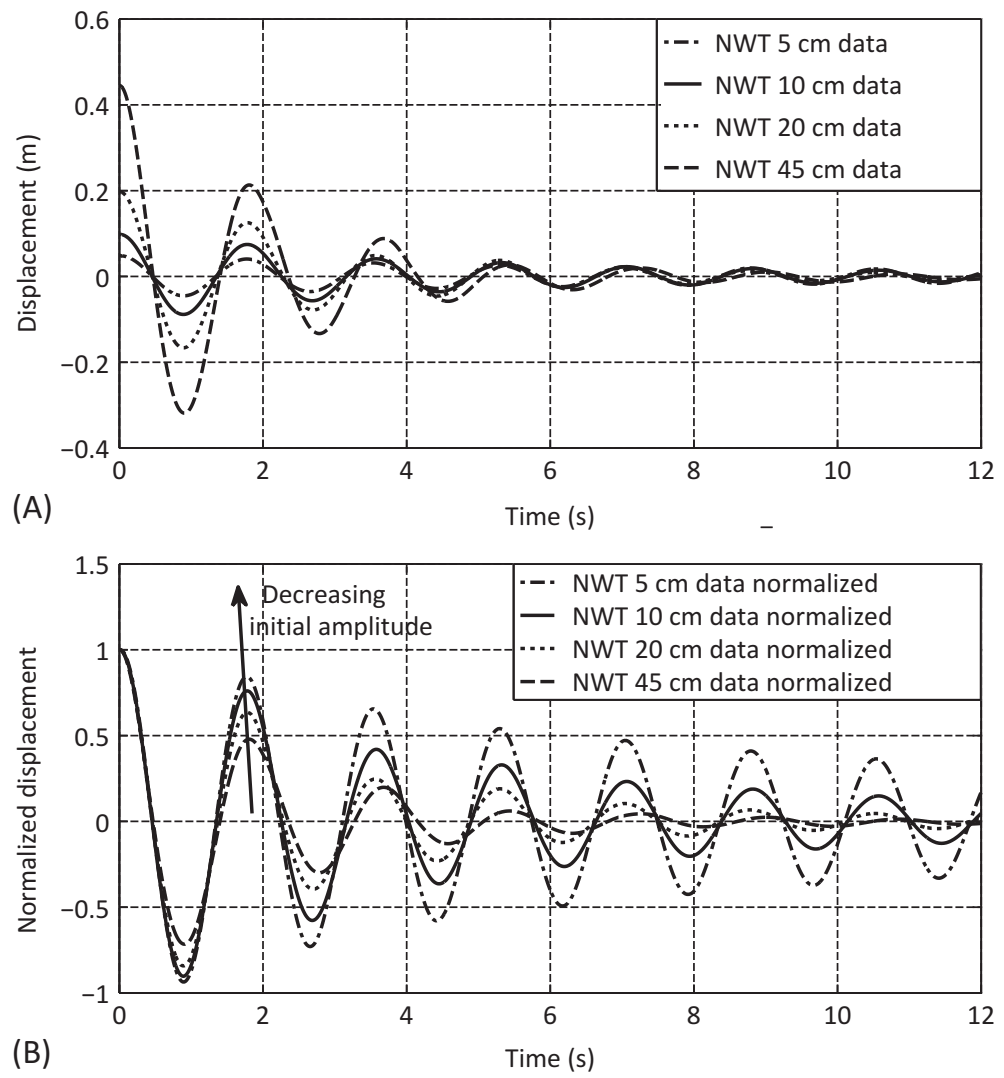

FIG. 7.11 (A) Simulated results from NWT free decay experiments. (B) Simulated results normalized against their initial position.

$$
\hat{\theta}=\operatorname{argmin} \sum_{i}\left|y_{N W T}\left(t_{i}\right)-y\left(t_{i}, \theta\right)\right|^{2} .
$$

The optimization problem in Eq. (7.11) is nonlinear in the parameters, with a strong sensitivity to the initial seed $\theta_{0}$, caused by an objective function with multiple local minima. To ensure that a good global solution is achieved, a concurrent search method is employed, which maintains a number of candidate solutions spread across the search space. In particular, the MATLAB implementation of a genetic algorithm is utilized as a solver.

Identifying linear models from the different NWT free decay data results in different parameter values. This can be summarized in Fig. 7.12, which shows the dominant poles for each model identified on different NWT data and for the model identified from the boundary element solver WAMIT (BEM-IM). Here, it can be seen that the magnitude of the real part of the conjugate poles decreases with the initial amplitude of the free decay data that the model was identified from. In fact, it can be seen that as the initial amplitude of the NWT identification data decreases, the poles converge on the result obtained from the BEM, which is based on the assumption of infinitesimally small oscillations. To further display this divergence from the BEM results as the amplitude of the NWT data increases, the radiation resistance and the added mass for each NWT identified model are calculated. Fig. 7.13A shows that the radiation resistance curves for the NWT identified models converge to the BEM-IM curve obtained from 


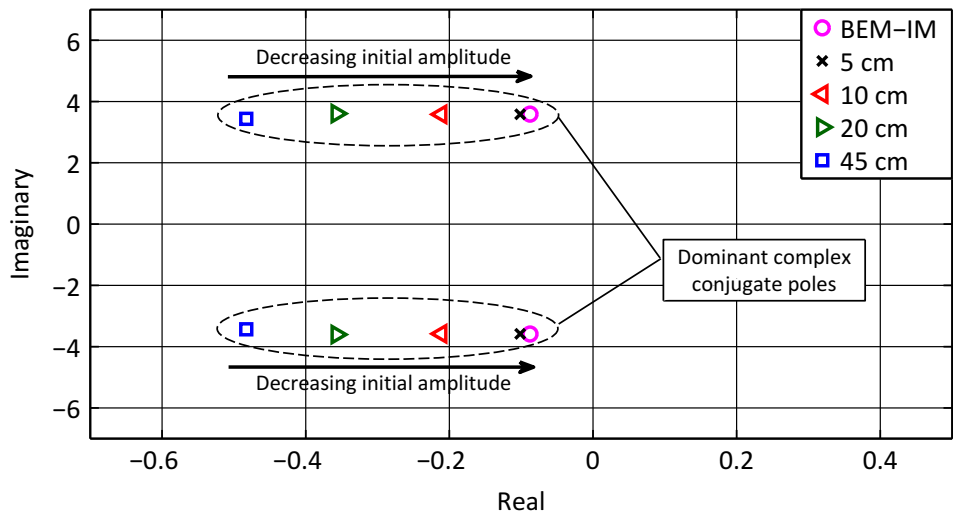

FIG. 7.12 Location on the complex-plane of the models' dominant poles.
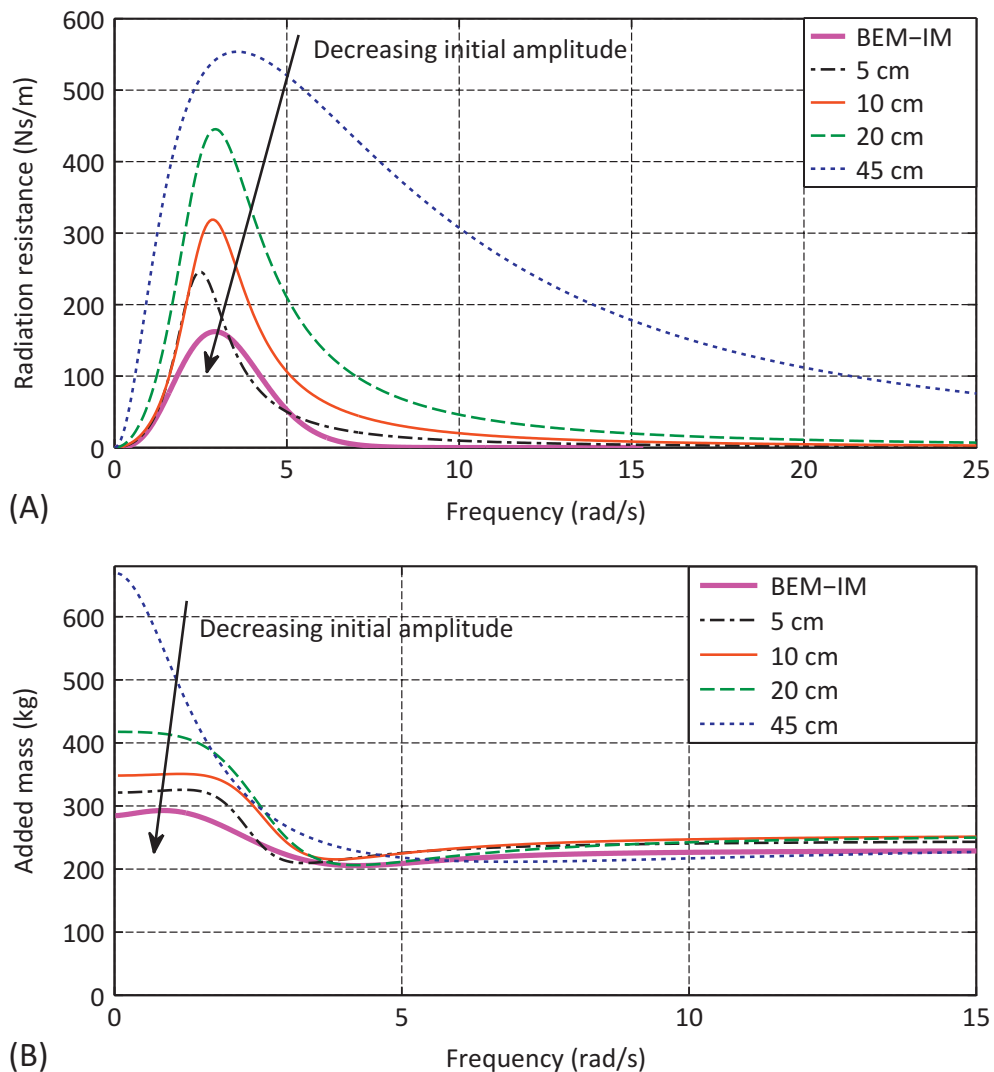

FIG. 7.13 (A) Radiation resistance and (B) added mass, both calculated by the BEM software WAMIT and compared against those identified from the NWT experiments. 


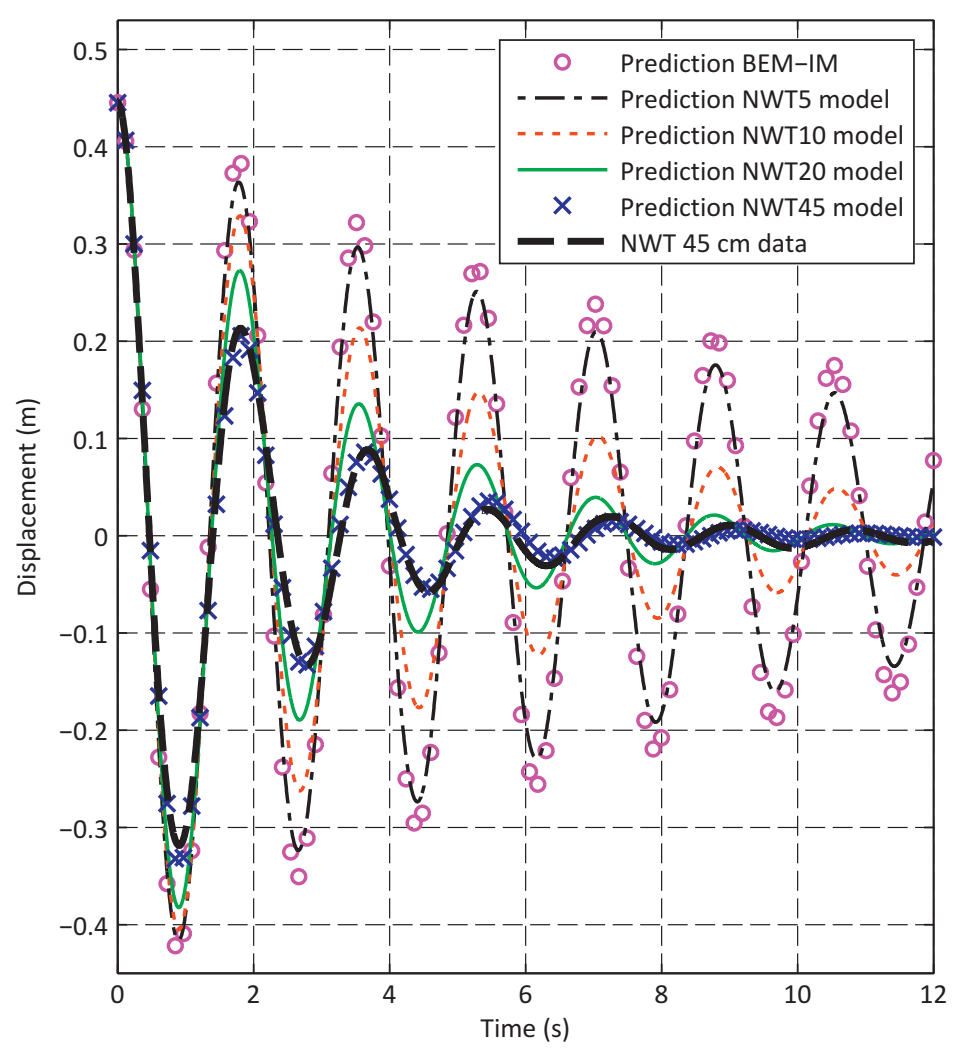

FIG. 7.14 Comparison of the different model predictions for an initial displacement of $45 \mathrm{~cm}$.
WAMIT, as the initial free decay displacement decreases. The radiation resistance is the only dissipative term in the identified linear models; therefore all of the different dissipative effects modelled by CFD (viscosity and vortex shedding, as well as wave radiation) are all encapsulated into this radiation resistance term. By decreasing the oscillation amplitude in the CFD simulations, the viscosity and vortex shedding becomes negligible, and the results converge on those obtained from BEM. A similar result is shown in Fig. 7.13B for the added mass.

The consequence of the inherent differences between the linear models is that they are representative of the operating region/amplitude that they are identified from, and then lose fidelity as they try to predict system behaviour away from this region. This is shown in Fig. 7.14, which plots the NWT data from the $45 \mathrm{~cm}$ free decay experiment as well as the free decay predictions of the different models, when given an initial amplitude of $45 \mathrm{~cm}$ (the number in the model names indicates the initial amplitude of the NWT data they were identified from). As expected, the NWT45 model predicts closest to the NWT experimental data, since it is the exact dataset the model was optimized to fit. The other models' predictions are progressively worse the further their initial amplitude is from the $45 \mathrm{~cm}$ point and the BEM-IM model has the worst fit, which is also expected, considering it is effectively linearized about an infinitesimally small deviation around the equilibrium position. 


\subsubsection{Case Study 2: Discrete-Time Models From Forced Oscillation}

This case study illustrates identifying a discrete-time model for the heave motion of a floating body in response to an input force (Fig. 7.4, Block B). Initially, a simple linear ARX model is considered and then the addition of a nonlinear static block is demonstrated to enable the model to capture nonlinear effects. Full details for this study can be found in Davidson et al. (2014).

The body's geometry is a vertical cone, which has strong nonlinearity in its restoring force, and is thus chosen to illustrate the capabilities of the different model structures in handling this effect. The cone has a diameter and height of $1 \mathrm{~m}$ and is orientated with its axis aligned vertically, with the base above the tip. The cone's relative density is 0.5 , resulting in a submerged draught of $0.8 \mathrm{~m}$. In this illustrative example, we consider the case where the body is subjected to a relatively low frequency $(0.125 \mathrm{~Hz})$ sinusoidal input force. The frequency was intentionally chosen to be low, to emphasize the static nonlinearity of the restoring force, by reducing other possible velocity dependent nonlinearities. The body was also initially displaced $30 \mathrm{~cm}$ above its equilibrium, allowing its free decay oscillation to be superimposed with the response to the input force.

For comparison, a linear CT model based on the Cummins equation is also evaluated (Cummins BEM model), using frequency domain hydrodynamic coefficients calculated using the BEM software WAMIT. The Cummins BEM model's linear restoring force parameter, $K$, is defined as the product of the water density, $\rho$, the gravitational constant, $g$, and the cone's free surface cross-sectional area at equilibrium, $S$, ie, $K=\rho g S$.

In Fig. 7.15A, are heave motion predictions by the NWT simulation, the ARX and the Cummins BEM models, when the sinusoidal input force has a relatively small amplitude $(240 \mathrm{~N})$. The output contains two parts, the transient due to the initial condition and the steady-state response to the harmonic input. It can be seen that, although the three predictions handle the transient part at the start slightly differently, they converge to the same result for the response to the input force. For these small amplitude conditions the linear models are shown here to work well.

A second simulation is then performed of the exact same experiment, except the amplitude of the input force is increased by a factor of four, to $960 \mathrm{~N}$ (Fig. 7.15B). Here we can see that the resulting body motions are much larger and that the performance of the three models diverges. The fully nonlinear NWT simulation exhibits an asymmetrical output, with respect to the equilibrium position, in response to the sinusoidal input, owing to the fact that the cone's geometry makes it harder to push into the water than out. It is not possible for the linear models to replicate this nonlinear behaviour.

To enable the linear ARX model to capture the nonlinearities in the floating body's motion, the model structure is extended by adding a nonlinear static block in series with the ARX model to construct a Hammerstein model and also in feedback to form a feedback block-oriented model (as in Fig. 7.8B). The static blocks for the Hammerstein and feedback block-oriented models are first identified using a prescribed motion experiment in the NWT, whereby the WEC is slowly moved through its full range of displacement while the corresponding hydrodynamic force is measured, Fig. 7.16A and B, respectively. If the WEC is moved slowly enough then all of the velocity and acceleration related effects (dynamic forces) will be negligible and the measured total force from the fluid will consist only of the position dependent effects, ie, the hydrostatic force. The hydrostatic force can then be evaluated as a function of the WEC displacement, Fig. 7.16C, and is then transformed to give the static curve $r()$ for the Hammerstein model (Fig. 7.16D) and $h()$ for the feedback block-oriented model (Fig. 7.16E). Also in Fig. 7.16D and $\mathrm{E}$ is the nonlinear static functions' fit to the NWT data. 

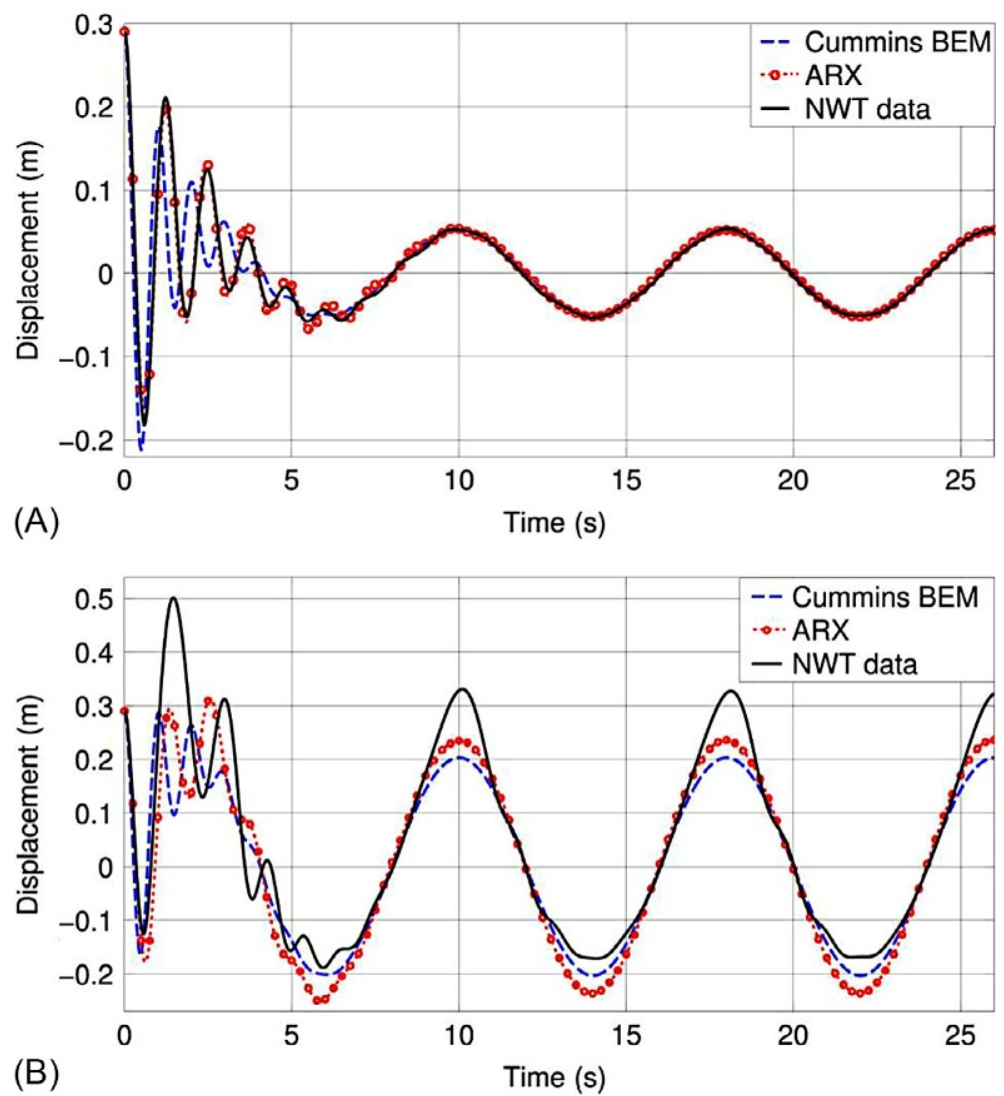

FIG. 7.15 NWT simulation results and linear models' predictions: (A) $240 \mathrm{~N}$ sinusoidal input force, and (B) $960 \mathrm{~N}$ sinusoidal input force.

The predictions of the identified nonlinear models compared to the NWT simulation are shown in Fig. 7.17. Here it can be seen that the nonlinear models outperform the linear models for the same case in Fig. 7.15B. The nonlinear models are able to replicate the asymmetrical steady-state response, and better able to reproduce the transient oscillations, particularly the feedback block-oriented model which does very well.

\subsubsection{Case Study 3: Discrete-Time Models From Input Waves}

This case study illustrates using discrete-time models to simulate the heave motion of a floating body subjected to input waves. Three different model structures are considered, the ARX, KGP, and ANN models. The models are trained on one set of NWT experimental data and then their performance validated on a second data set.

The input waves used in this case study are designed to replicate a sea spectrum. Both the training and validation experiments use multisine wave signals as input, consisting of 100 equally spaced frequencies, in the range $0.005-0.995 \mathrm{~Hz}$, with randomly assigned phases and amplitudes determined from a JONSWAP spectrum with significant wave height $H_{s}=0.5 \mathrm{~m}$, peak period $T_{p}=$ $6 \mathrm{~s}$ and peakiness $\gamma=2$. Each experiment is $600 \mathrm{~s}$ in length and records the FSE at the body's centre of 

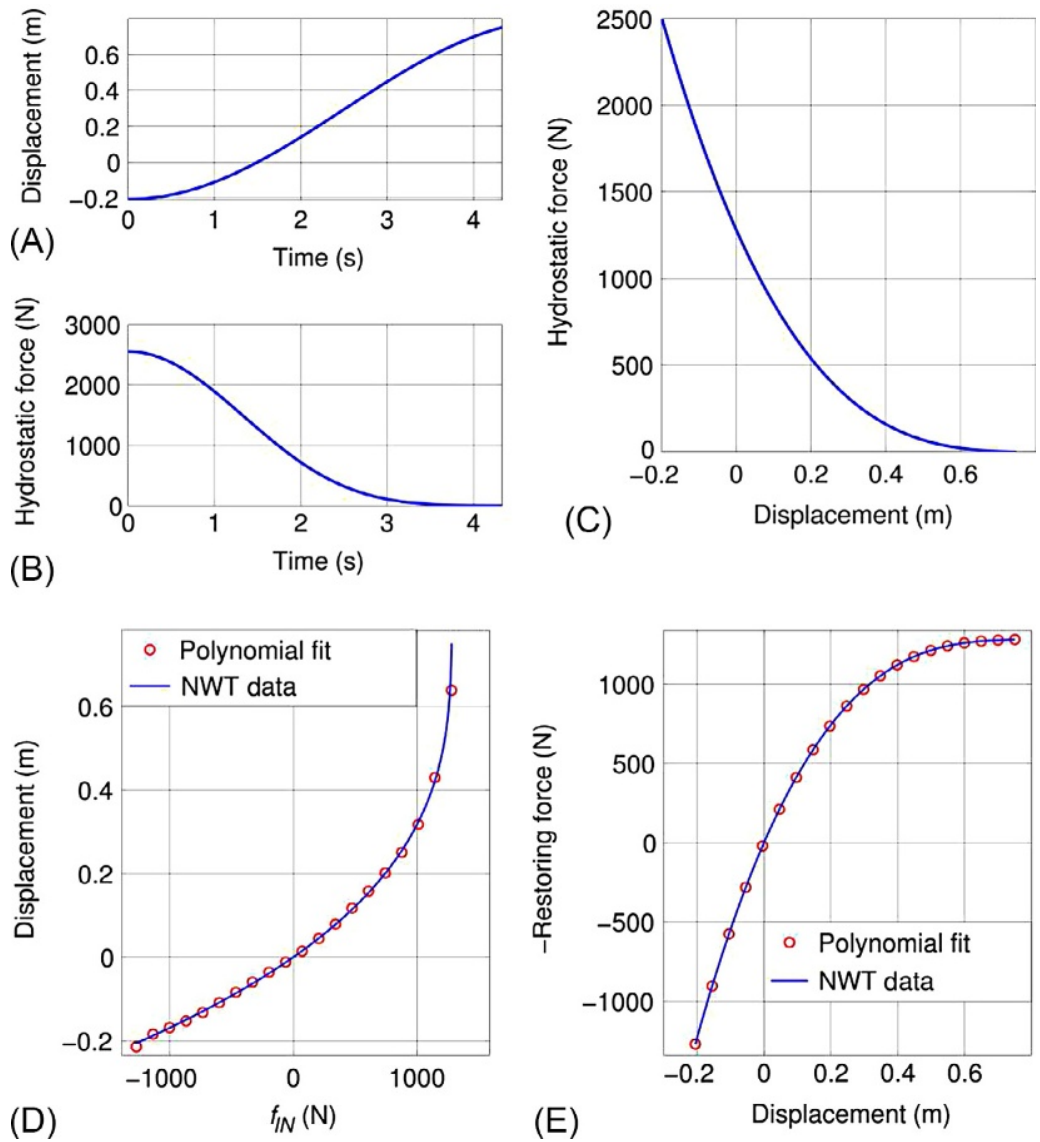

FIG. 7.16 (A) Prescribed motion experiment: WEC displacement. (B) Prescribed motion experiment: hydrostatic force. (C) The hydrostatic force as a function of the WEC displacement. (D) Static curve $r($ ) for the Hammerstein model. (E) Static curve $h()$ for the feedback block-oriented model.

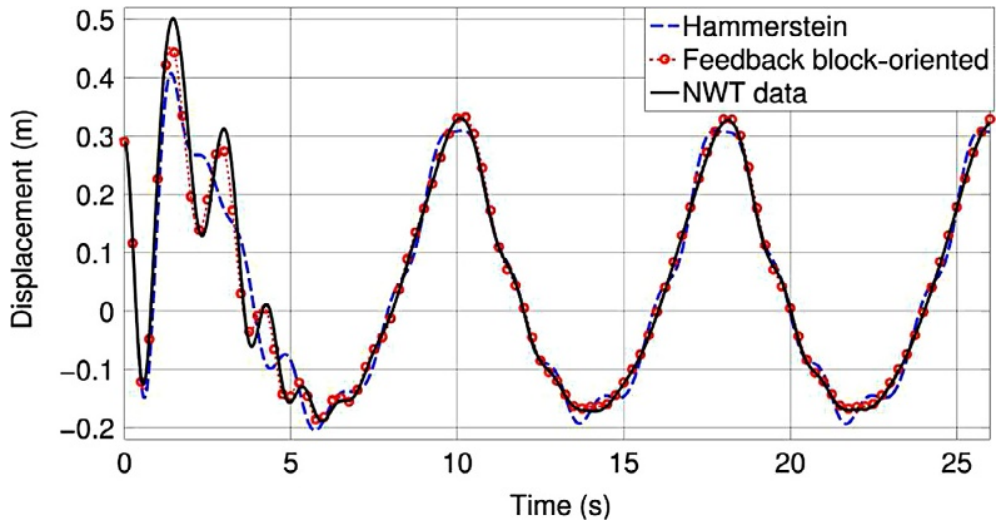

FIG. 7.17 NWT simulation results and nonlinear models' predictions: $960 \mathrm{~N}$ sinusoidal input force. 
mass as the model input and the heave motion of the body as the output, following the procedure depicted in Fig. 7.4.

Using the strategies described in Section 7.4.2.1, the input delay time, $n_{d}=-7$, the dynamical orders, $n_{a}=8$ and $n_{b}=2$, have been identified for the three models. Fig. 7.18A shows the result of the trial-and-error process to calculate $n_{d}$, where it is possible to see that the minimum occurs for $n_{d}=-7$ (noncausal ARX model). Fig. 7.18B plots the loss function for different values of $n_{a}$, from which it can be seen there is not any significant improvement for $n_{a}>8$.

For the KGP structure, the presence of the cross-product terms resulted in an instability in the identified models. For this reason, the cross-product terms in the model of Eq. (7.2) were removed. A polynomial order of two for the KGP model ( $p=2$ ) has been identified, by observing that bigger values of $p$ improve the training fitting but degrade the quality of the validation fitting (overfitting). For the ANN structure, a good compromise between complexity and accuracy of the model has been found utilizing $n_{1}=10$ and $n_{2}=10$.

An example of the models' ability to simulate the motion of the device is shown in Fig. 7.19, where (A) shows the performance of the models compared with the NWT simulation for the training experiment and (B) for the validation
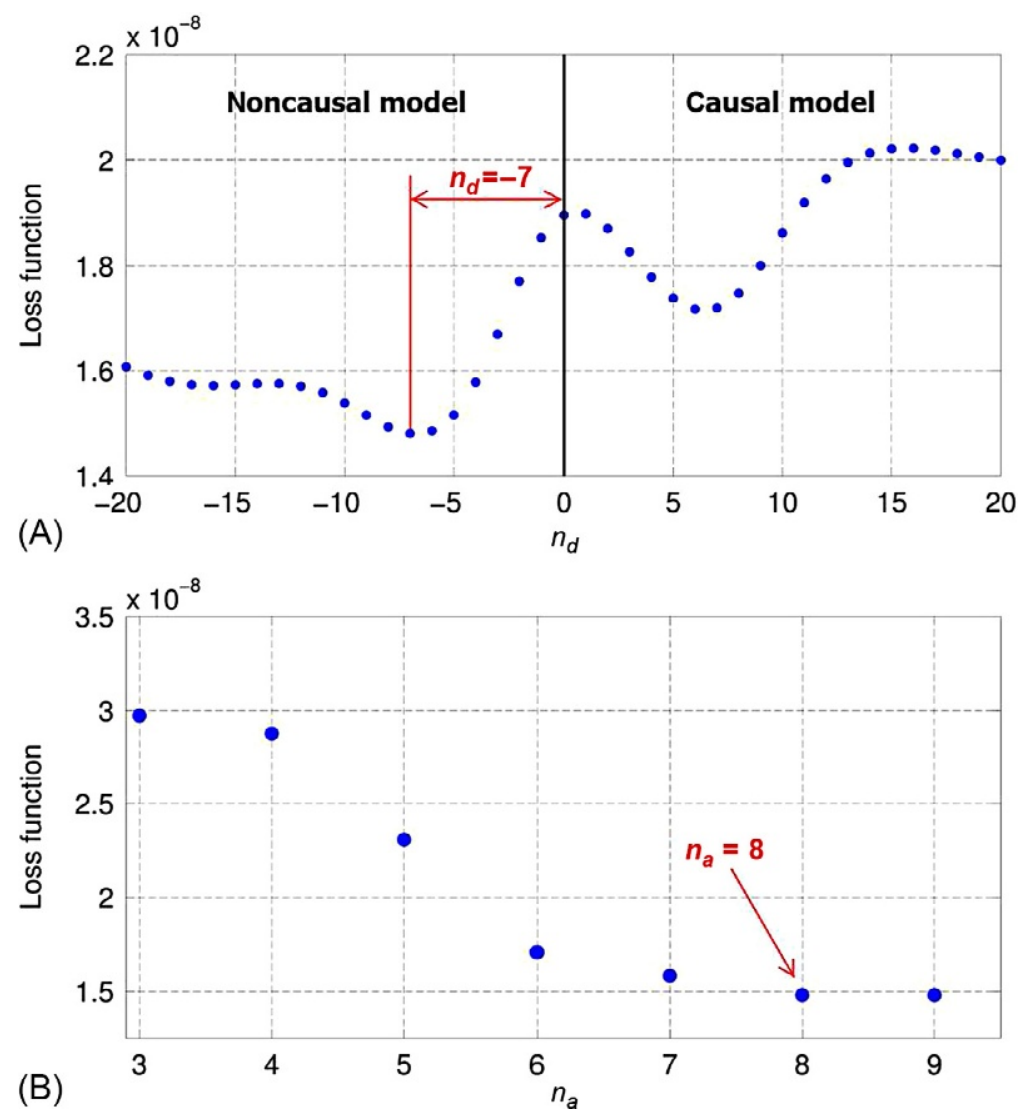

FIG. 7.18 (A) Determination of $n_{d}=-7$ for the models. (B) Determination of the dynamical order $n_{a}=8$ for the models. 

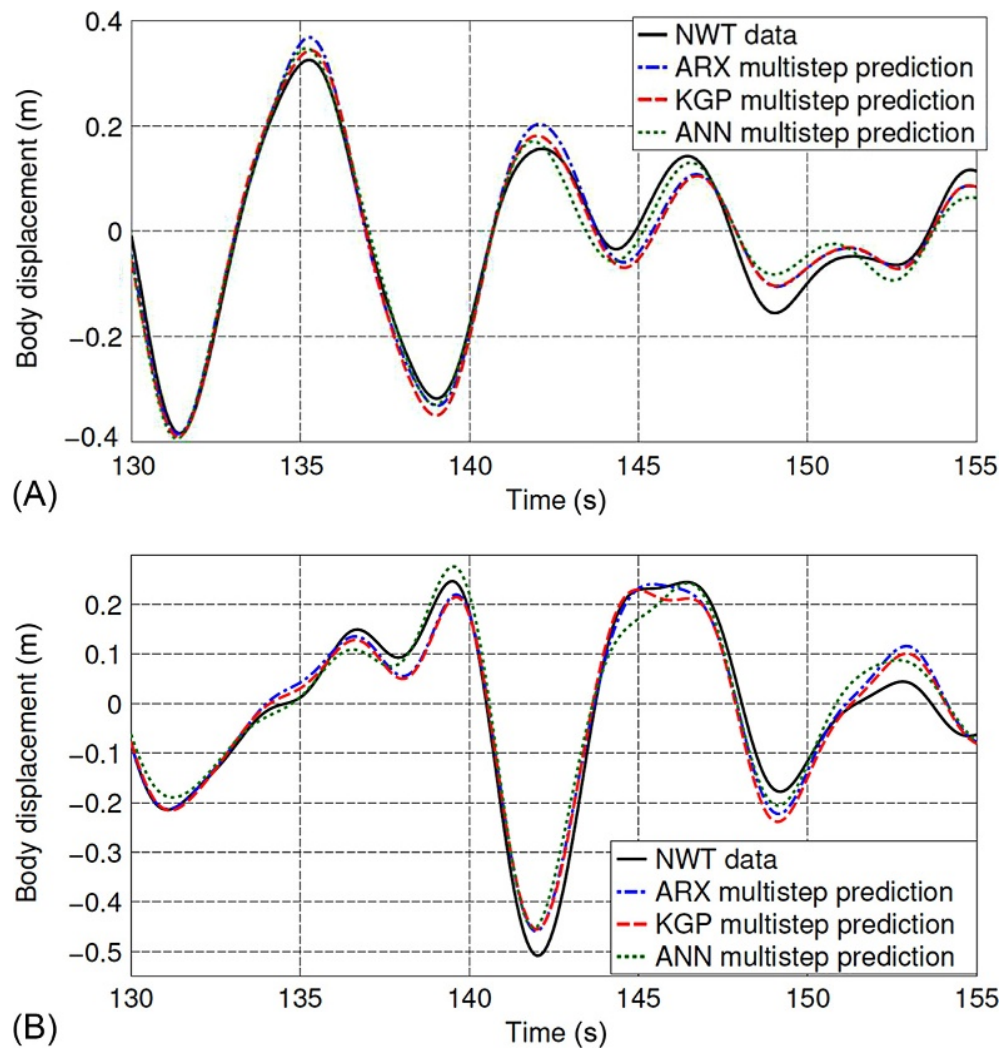

FIG. 7.19 (A) Identified models' performances on the training experiment. (B) Identified models' performances on the validation experiment.

experiment. In both training and validation cases, all three models are seen to replicate the device motion quite well.

The normalized root mean-squared error (NRMSE) between the models' predictions, $\hat{y}(k)$, and the NWT data, $y(k)$, is used to quantify the model's performances, where $N R M S E=\left(\sqrt{\sum_{k}|y(k)-\hat{y}(k)|^{2}} / \sqrt{\sum_{k}|y(k)|^{2}}\right)$.

The NRMSE values for the ARX model are 0.1721 on the training data and 0.1793 on the validation; the KGP model scored 0.1713 training and 0.1787 validation; and the ANN model 0.1671 training and 0.2529 validation.
These results show little difference in the performance of the linear ARX model compared to the two nonlinear models, indicating that the floating body's motion does not exhibit much nonlinear behaviour for the geometry and wave conditions chosen in this case study. The ARX and KGP models maintain similar performances for training and validation, indicating a good selection of model order and parameter identification. The ANN model was best able to fit the training data, but its performance is seen to degrade considerably between training and validation, demonstrating the difficulties associated with the nonlinear identification of neural networks. 


\subsection{LIMITATIONS}

The main limitations of this modelling approach are based on the limitations of the data used to identify the models: the models can only ever be as accurate as the data they are identified from. Data from physical experiments can suffer from measurement noise and error, scaling effects, mechanical friction, and wave reflections. The data quality from NWT experiments are reliant on the accuracy of the CFD simulations.

The use of black-box models, introduced in this chapter, are limited by the information contained in the data they are trained on. These black-box models simply reproduce the experimental output data, given the same stimulus, but the internal model structure bears no resemblance to the physical world and, therefore, they have no guarantee on extrapolating well when subjected to input conditions outside of the frequency and amplitude ranges they were trained on.

For models whose parameter identification results in nonconvex optimization problems, there is no guarantee of achieving the global minimum solution.

\subsection{SUMMARY}

- Model identification from recorded data can produce accurate models of WECs, provided that the data is of a sufficiently high quality.

- A range of model structures and identification techniques can be applied to WEC models, each with specific advantages and disadvantages.

- The model structure should be chosen so that it captures the relevant linear and nonlinear hydrodynamic effects.

- The accuracy of the models are dependent on the quality of the recorded data.

- Identification experiments, used to generate the recorded data, should be designed to ensure the data has good coverage in the expected operational conditions.
- Typical identification experiments include free decay tests, force oscillation tests, prescribed motion tests and wave excitation tests.

\section{References}

Armesto, J.A., Guanche, R., Iturrioz, A., Vidal, C., Losada, I.J., 2014. Identification of state-space coefficients for oscillating water columns using temporal series. Ocean Eng. 79, 43-49.

Babarit, A., Laporte-Weywada, P., 2009. On the numerical modelling of the nonlinear behaviour of a wave energy converter. In: ASME 2009 28th International Conference on Ocean, Offshore and Arctic Engineering.

Bai, E.W., 2003. Decoupling the linear and nonlinear parts in Hammerstein model identification. Automatica 40, 671-676.

Bhinder, M.A., Babarit, A., Gentaz, L., Ferrant, P., 2011. Assessment of viscous damping via 3d-CFD modelling of a floating wave energy device. In: Proceedings of the 9th European Wave and Tidal Energy Conference, Southampton, UK.

Bhinder, A., Babarit, M.A., Gentaz, L., Ferrant, P., 2012. Effect of viscous forces on the performance of a surging wave energy converter. In: The Twenty-second International Offshore and Polar Engineering Conference, pp. 545-549.

Bonfiglio, L., Brizzolara, S., Chryssostomidis, C., 2011. Added mass and damping of oscillating bodies: a fully viscous numerical approach. In: Recent Advances in Fluid Mechanics, Heat \& Mass Transfer and Biology.

Davidson, J., Giorgi, S., Ringwood, J., 2013. Linear parametric hydrodynamic models based on numerical wave tank experiments. In: 10th European Wave and Tidal Energy Conference (EWTEC), Aalborg.

Davidson, J., Giorgi, S., Ringwood, J., 2014. Numerical wave tank identification of nonlinear discrete-time hydrodynamic models. In: Proc. 1st Int. Conf. on Renewable Energies Offshore (Renew 2014), Lisbon.

Davidson, J., Cathelain, M., Guillemet, L., Le Huec, T., Ringwood, J., 2015. Implementation of an OpenFOAM numerical wave tank for wave energy experiments. In: 11th European Wave and Tidal Energy Conference (EWTEC), Nantes.

Davidson, J., Giorgi, S., Ringwood, J.V., 2015. Linear parametric models for ocean wave energy converters identified from numerical wave tank experiments. Ocean Eng. 103, 31-39.

Giorgi, S., Davidson, J., Ringwood, J.V., 2015. Identification of nonlinear excitation force kernals using numerical wave tank experiments. In: 11th European Wave and Tidal Energy Conference (EWTEC), Nantes. 
Giri, F., Bai, E.W., 2010. Block-Oriented Nonlinear System Identification. Springer, Berlin, Heidelberg, Germany.

Guérinel, M., Alves, M., Sarmento, A., 2011. Nonlinear modelling of the dynamics of a free floating body. Proceedings of the 9th European Wave and Tidal Energy Conference, Southampton, UK.

Lawson, M., Yu, Y.H., Nelessen, A., Ruehl, K., Michelen, C., 2014. Implementing nonlinear buoyancy and excitation forces in the WEC-SIM wave energy converter modeling tool. In: ASME 2014 33rd International Conference on Ocean, Offshore and Arctic Engineering.

Ljung, L., 1999. System Identification (2nd Ed.): Theory for the User. Prentice Hall PTR, Upper Saddle River, NJ, USA.

Ljung, L., 2010. Perspectives on system identification. Ann. Rev. Control 34 (1), 1-12.

Ljung, L., 2013. MATLAB System Identification Toolbox. MathWorks, Natick, MA, USA.

Nelles, O., 2001. Nonlinear System Identification: From Classical Approaches to Neural Networks and Fuzzy Models. Springer, Berlin, Heidelberg, Germany.

Nievergelt, Y., 2000. A tutorial history of least squares with applications to astronomy and geodesy. J. Comput. Appl. Math. 121 (1-2), 37-72.
Pearson, R.K., Pottmann, M., 2000. Gray-box identification of block-oriented nonlinear models. J. Process Control 10, 301-315.

Pintelon, R., Schoukens, J., 2012. System Identification-A Frequency Domain Approach, 2nd edition Wiley, Hoboken, NJ.

Rao, G., Unbehauen, H., 2006. Identification of continuoustime systems. Proc. IEE-Control Theory Appl. 153, 185-220.

Ringwood, J.V., Davidson, J., Giorgi, S., 2015. Optimising numerical wave tanks tests for the parametric identification of wave energy device models. In: Proc. of the 34th Int. Conf. on Ocean, Offshore and Arctic Engineering (OMAE 2015).

Tanizawa, K., 2000. The state of the art on numerical wave tank. In: Proceedings of 4th Osaka Colloquium on Seakeeping Performance of Ships.

Zurkinden, A.S., Ferri, F., Beatty, S., Kofoed, J.P., Kramer, M., 2014. Non-linear numerical modeling and experimental testing of a point absorber wave energy converter. Ocean Eng. 78, 11-21. 